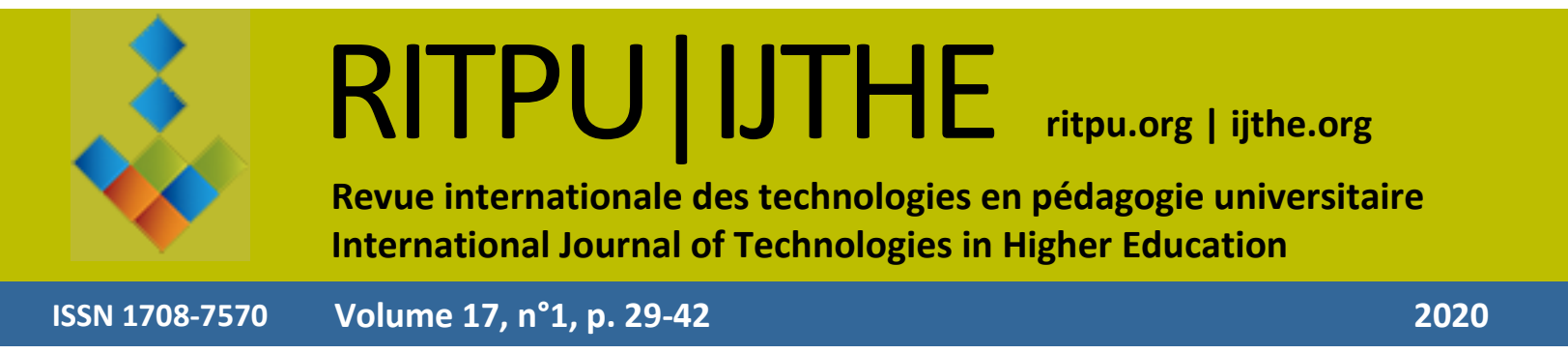

\title{
Physique, téléphone intelligent et technologie d'impression 3D : étude de cas sur la transition numérique dans l'enseignement des sciences
}

Chris Isaac LARNDER chrisisaac.larnder@johnabbott.qc.ca

Collège John Abbott

https://doi.org/10.18162/ritpu-2020-v17n1-09

Faïza NEBIA

FNEBIA@cvm.qc.ca

Cégep du Vieux Montréal

Margaret LIVINGSTONE
m.livingstone@marianopolis.edu
Collège Marianopolis
Shiwei HUANG
shiwei.huang@johnabbott.qc.ca
Collège John Abbott

Collège John Abbott

\section{Résumé}

Nous décrivons les stades du développement technologique dans le cadre d'un projet de quatre ans visant à adapter l'enseignement de la physique au collégial aux réalités de l'ère numérique. Tirant parti de multiples innovations complémentaires, ce projet nous sert d'étude de cas en vue d'acquérir une compréhension plus générale du déroulement des transitions technologiques dans un cadre pédagogique et, plus particulièrement, dans l'enseignement en sciences et en génie.

Nous examinons le cycle de vie complet d'un projet de changement technologique, y compris l'évaluation des défis à plus long terme tels que l'acquisition de nouvelles compétences par le personnel enseignant et de soutien, et la nécessité pour les établissements d'enseignement participants d'investir dans l'équipement. Notre analyse utilise comme cadre de référence le récent Plan d'action numérique du Québec, afin d'illustrer les avantages indirects qu'un projet fondé sur la technologie peut tirer d'investissements publics qui ciblent l'ensemble du réseau collégial.

\section{Mots-clés}

Numérique, enseignement, formation enseignante, développement enseignant, cheminement professionnel 


\section{Introduction}

\section{L'enseignement des sciences dans un paysage numérique}

De plus en plus, les étudiants d'aujourd'hui sont immergés dans un paysage numérique. Leur principale source d'expériences est médiée par un écran mobile ou un moniteur de portable plutôt que par des interactions directes avec le monde physique (Alvermann, 2004; Morley, 2001). Cette nouvelle réalité a des conséquences de taille pour la pédagogie scientifique, et plus particulièrement pour l'enseignement de la physique (Crompton et al., 2016; Strimel et Grubbs, 2016).

Un des aspects fondamentaux de l'enseignement des sciences est la création de liens entre les théories générales et l'application de ces théories à certains phénomènes de la vie quotidienne. Pour maintenir l'engagement des étudiants en sciences, les enseignants doivent renouveler leur répertoire d'exemples classiques en y ajoutant des cas tirés de l'ère numérique, qui ont davantage de pertinence dans le monde nouveau où vivent les étudiants d'aujourd'hui.

Pour ces étudiants, l'expérience de la physique au quotidien n'a plus guère de rapports avec un plan incliné ou avec la trajectoire d'une balle de baseball; le basculement automatique du mode portrait au mode paysage sur l'écran de leur téléphone leur parle davantage. De fait, ce type de comportements du téléphone intelligent sert de point de départ pour le genre de projet de transition numérique que nous décrirons ci-après.

\section{La transformation du contenu et la transformation de la transmission du contenu}

La révolution numérique a produit un large éventail d'outils de technologies de l'information qui transforment notre façon de communiquer avec les étudiants et de leur transmettre du contenu, quelle que soit la matière à l'étude. La transformation du mode de transmission fait déjà l'objet de maintes discussions dans les publications sur la recherche en éducation (Blazer, 2008, p. 34; Schindler et al., 2017), mais elle est indépendante de la transition numérique dont il est question ici. En effet, elle influe sur le mode de transmission du contenu, tandis que nous nous intéressons ici à une mutation du contenu lui-même. Dans le premier cas, il s'agit d'adapter, puis d'exploiter des outils rendus possibles par la technologie; dans le second, comme nous allons l'expliquer, on intègre au curriculum une compréhension de la technologie numérique et des paradigmes de traitement des données qui lui sont associés.

\section{La physique classique à la rencontre du monde numérique}

L'un des principaux obstacles à la transition numérique vient du fait que le contenu du curriculum constitue - pour emprunter un terme à la théorie des jeux - «un jeu à somme nulle»: si nous ajoutons de nouveaux contenus ayant des liens avec les technologies numériques, une partie des contenus traditionnels doit leur céder la place. Notre stratégie consiste donc à concevoir des exemples de comportements du monde numérique qui illustrent des concepts scientifiques classiques. Ainsi, le contenu traditionnel demeure intact; seuls les exemples traditionnels sont remplacés par d'autres, issus de l'ère numérique.

Notre défi est donc de trouver, parmi les idées et les concepts de la technologie numérique, des exemples qui illustrent des concepts classiques de la physique. C'est exactement ce que fait le projet visé par notre étude de cas : concevoir avec soin des activités où les étudiants examinent un comportement du téléphone intelligent qui illustre un principe fondamental de la physique. 
Le potentiel général qu'a le téléphone intelligent de bonifier l'expérience pédagogique a fait l'objet de maintes analyses (Buck et al., 2013, p. 11; Langan et al., 2016), y compris dans cette revue (Mammadova, 2018). La communauté de la recherche sur l'enseignement de la physique s'y intéresse depuis un certain temps : des essais antérieurs ont été réalisés avec des appareils portatifs tels que la Wiimote de Nintendo (Hochberg et al., 2016), mais la grande polyvalence du téléphone intelligent (Arribas et al., 2015; Monteiro et Martí, 2016) et de la tablette (Egri et Szabó, 2015) a rapidement suscité davantage d'intérêt.

Le projet que nous allons analyser ici tire parti de ces initiatives. En plus d'avoir lui aussi été décrit dans diverses publications internationales (Hinrichsen et Larnder, 2018; Larnder, 2019a), il a fait l'objet d'une vaste promotion à l'échelle locale (Larnder et Portelance, 2019a, 2019b) et, dans certains cas (Larnder, 2019b; Moon, 2018), d'une publication dans les deux langues officielles. Ce projet propose une série d'activités de laboratoire fondées sur l'accéléromètre, un capteur dont sont dotés les téléphones intelligents de la génération actuelle. Une autre technologie de l'ère numérique, celle de l'impression $3 \mathrm{D}$, est mise à profit pour construire les appareils de laboratoire utilisés dans ces activités.

\section{Le Plan d'action numérique du Québec}

Le gouvernement du Québec reconnaît depuis longtemps la nécessité de stimuler et de soutenir le changement systémique dans son système éducatif. Plus particulièrement, au niveau collégial, il administre plusieurs programmes ${ }^{1}$ qui sont de plus en plus orientés sur l'habileté numérique. Notre étude de cas, par exemple, en est à sa quatrième année de financement par l'entremise de programmes de ce genre.

Le gouvernement du Québec a récemment déployé son Plan d'action numérique en éducation et en enseignement supérieur (MEES, 2018), un projet pluriannuel ambitieux visant à réaliser une transition numérique dans l'ensemble du système éducatif québécois. Le plan d'action distingue trois grandes catégories d'activités et d'investissements (tableau 1).

\section{Tableau 1}

Grandes orientations du Plan d'action numérique

\begin{tabular}{|c|c|}
\hline Orientation & Description \\
\hline 1 & Soutenir le développement des compétences numériques des jeunes et des adultes \\
\hline 2 & $\begin{array}{l}\text { Exploiter le numérique comme vecteur de valeur ajoutée dans les pratiques d'enseignement } \\
\text { et d'apprentissage }\end{array}$ \\
\hline 3 & $\begin{array}{l}\text { Créer un environnement propice au déploiement du numérique dans l'ensemble du } \\
\text { système éducatif }\end{array}$ \\
\hline
\end{tabular}

Globalement, ce cadre est très complet, de sorte qu'il peut servir de liste de contrôle, même pour les parties prenantes qui évaluent des projets non financés directement par lui, tel celui qui nous occupe ici. La dernière orientation du tableau, qui représente une grande partie du budget, a trait aux investissements infrastructurels, dont certains seraient particulièrement susceptibles de

1. Le Programme de recherche et d'expérimentation pédagogiques (PREP); le Programme d'aide à la recherche sur l'enseignement et l'apprentissage (PAREA); l'Entente Canada-Québec relative à l'enseignement dans la langue de la minorité et à l'enseignement des langues secondes (ECQ); le Programme de la recherche collégiale du Fonds de recherche du Québec - Nature et technologies (FRQNT); Programme de recherche sur la persévérance et la réussite scolaires (PRPRS). 
contribuer à l'atteinte des objectifs du projet à l'étude en matière de développement des technologies numériques, comme nous l'expliquerons plus loin.

\section{Les activités de laboratoire à l'ère numérique}

Le projet à l'étude avait pour objectifs initiaux de développer un nouvel ensemble d'activités de laboratoire mettant en jeu des appareils et des phénomènes propres à l'ère numérique. Ces activités doivent respecter une condition : renforcer l'apprentissage et l'intégration des principes classiques de la physique énoncés dans le curriculum collégial existant. Les essais devaient être réalisés itérativement, avec un seul étudiant à la fois, jusqu'à ce que l'on considère que les activités sont prêtes à utiliser en classe.

\section{L'accéléromètre et le téléphone intelligent}

L'accéléromètre a rapidement été reconnu comme un type de dispositif dont l'exploration par les étudiants était prometteuse. Le concept d'accélération est omniprésent en physique, mais les étudiants ont souvent de la difficulté à le maîtriser; de plus, dans la plupart des activités de laboratoire, les valeurs de l'accélération découlent uniquement de calculs effectués sur des données relatives à la position des objets. Comme l'accéléromètre produit une mesure directe de l'accélération, il a le potentiel de rendre ce concept plus accessible aux étudiants. L'accéléromètre, un capteur qui mesure l'accélération, est d'usage courant dans les appareils mobiles tels que le téléphone intelligent et les dispositifs de monitorage du sommeil et de la condition physique.

Grâce aux avancées récentes dans le domaine des capteurs pour microsystèmes électromécaniques (micro-electromechanical systems - MEMS) (Gardner et al., 2001, chap. 8), ces dispositifs sont maintenant de petite taille, peu coûteux et assez précis pour produire des résultats fiables. Un large éventail d'accéléromètres autonomes ont été acquis et soumis à des tests mettant à l'épreuve leur fiabilité, leurs configurations possibles et leur utilisabilité dans le cadre d'un flux de production.

Les téléphones intelligents contiennent eux aussi un capteur accélérométrique, et on trouve de plus en plus d'applications permettant de consulter et de recueillir les données produites par ce dispositif. Au terme de la première année d'essais, il est devenu évident que les applis pour téléphone intelligent des plateformes Android et iOS étaient fiables et avaient la capacité de soutenir un flux de travaux relativement simple. Le téléphone intelligent est rapidement devenu l'appareil de choix pour les activités de laboratoire. L'engagement des étudiants s'enrichissait ainsi d'un élément supplémentaire : la fierté et la fascination d'utiliser un appareil si familier à une fin tout à fait nouvelle.

\section{La transition entre les modes portrait et paysage}

La première activité prometteuse exploite la transition bien connue qui se fait automatiquement entre les modes d'affichage «portrait» et «paysage» lorsqu'on change l'orientation d'un téléphone intelligent. Cette transition se produit notamment lors du visionnement de photos ou de textos. Les étudiants ont pour consigne de mesurer avec précision l'angle critique auquel cette transition se produit et d'interpréter l'extrant correspondant de l'accéléromètre, qui permet à l'appareil de détecter sa propre orientation. En examinant les données de l'accéléromètre, les étudiants déduisent l'angle critique qu'ils ont mesuré auparavant par d'autres moyens. 
Cette activité est un substitut numérique à l'exemple classique du plan incliné. Le concept fondamental de la physique qui entre en jeu est le même : la décomposition du vecteur gravitationnel en composants le long des axes d'un cadre de référence incliné, en l'occurrence celui du téléphone intelligent. Entre aussi en ligne de compte l'aptitude à comprendre la différence entre un système de coordonnées global et un système de coordonnées local, ainsi qu'à déterminer l'angle d'un vecteur décrit par ses composantes (tableau 2).

\section{Le téléphone tournant}

Dans une deuxième activité de laboratoire fructueuse, les étudiants sont invités à faire tourner leur téléphone sur lui-même et à examiner les données de l'accéléromètre. Cette fois, l'engagement des étudiants est stimulé par le vague sentiment de danger associé à la vision de leur cher téléphone animé d'une rotation rapide. Dans ce cas, les données de l'accéléromètre démontrent le principe de l'accélération centripète, une quantité qui existe même lorsque la vitesse de rotation d'un objet sur lui-même est uniforme.

Les étudiants en viennent à la constatation suivante : quelle que soit la position du téléphone sur la surface tournante, le vecteur de l'accélération pointe toujours vers le centre de rotation. De plus, ils peuvent réaliser un authentique exercice de rétro-ingénierie numérique. En regroupant les résultats obtenus à partir de plusieurs positions, ils arrivent à situer exactement l'emplacement du capteur accélérométrique dans le corps du téléphone. Ensuite, en consultant le schéma du circuit de leur modèle de téléphone, ils obtiennent une confirmation visuelle du résultat obtenu.

\section{Tableau 2}

Propriétés de deux exemples d'activités de laboratoire

\begin{tabular}{lcll}
\hline Titre & Concept classique & Compétences & $\begin{array}{c}\text { Appareil produit par } \\
\text { impression 3D }\end{array}$ \\
\hline $\begin{array}{l}\text { Étude du plan incliné } \\
\text { à l'aide d'un } \\
\text { téléphone intelligent } \\
\text { et du TiltTray }\end{array}$ & Plan incliné & $\begin{array}{l}\text { Gravité } \\
\text { Forces normales }\end{array}$ & $\begin{array}{l}\text { Le TiltTray } \\
\text { (plateau inclinable) }\end{array}$ \\
\hline $\begin{array}{l}\text { Mouvement } \\
\text { circulaire d'un } \\
\text { téléphone intelligent } \\
\text { sur le SpinFrame }\end{array}$ & Accélération & Systèmes de coordonnées local et global & Accélération centripète \\
\hline
\end{tabular}

\section{L'équipement produit par impression 3D}

Chaque activité de laboratoire nécessite certains appareils. La conception et le choix des matériaux de construction - des considérations importantes en elles-mêmes - sont souvent négligés lors du développement d'une activité de laboratoire. La technologie de l'impression 3D est une option de plus en plus attrayante: le coût total d'acquisition et d'utilisation des imprimantes 3D diminue progressivement, et le coût en matériel des objets imprimés est très bas (Zwicker et al., 2015). Cette technologie nous permet aussi de concevoir un prototype, de l'utiliser et de l'améliorer d'une façon itérative, ce qui a des conséquences importantes pour la pérennité du projet à l'étude. Enfin, la possibilité d'imprimer un appareil à partir d'un fichier électronique est attrayante et facilite les échanges avec les collègues d'autres établissements. 
L'expérience portrait-paysage a nécessité le développement d'un plateau inclinable appelé le TiltTray (figure 1). Ce plateau peut accueillir des téléphones intelligents de formes variées, et son angle d'inclinaison est réglable. Le rapporteur qui y est intégré permet à l'utilisateur de mesurer cet angle avec précision.

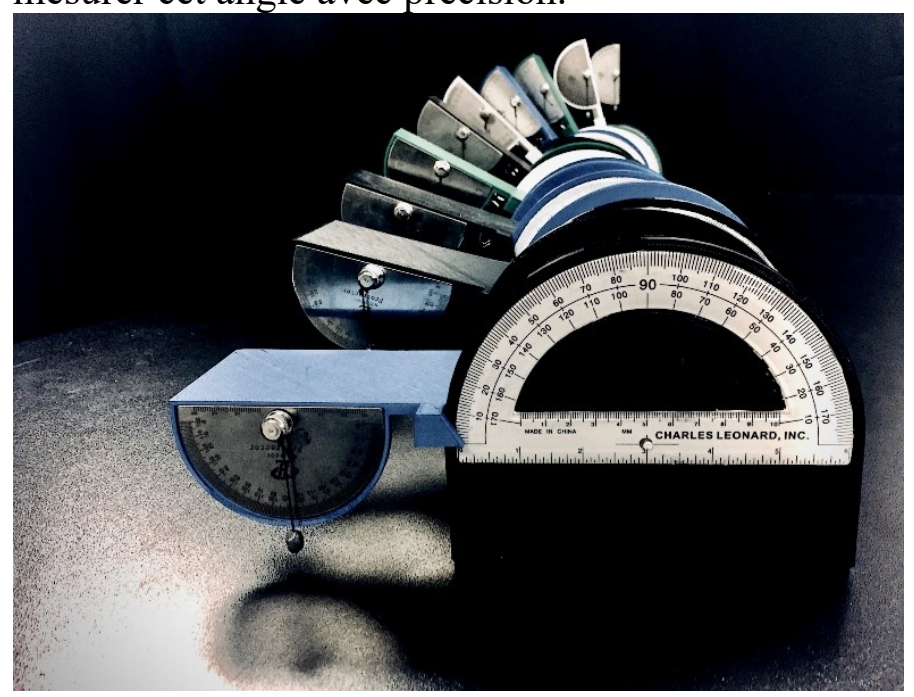

\section{Figure 1}

Ensemble de plateaux inclinables TiltTray produits par impression 3D

L'expérience du téléphone tournant a donné lieu au développement d'un autre appareil, le SpinFrame. On pose sur un cadre rectangulaire de $216 \mathrm{~mm}$ (8,5 po) sur $279 \mathrm{~mm}$ (11 po) une feuille de papier de format lettre, sur laquelle on place un téléphone intelligent. Le cadre empêche le téléphone de glisser à l'extérieur de la surface tournante et donne des positions de référence qui serviront à prendre des mesures. Le cadre est déposé sur le plateau d'une table tournante adaptée aux fins de l'expérience.

Ces deux appareils sont en fait des objets faits de «médias mixtes ». Le TiltTray est muni d'un rapporteur d'angle traditionnel, collé en place sur la surface produite par impression $3 \mathrm{D}$, tandis que le SpinFrame est doté d'une base motorisée, que l'on retrouve dans le commerce sous la forme d'un tourne-disque bon marché. Un certain assemblage est donc requis, d'où la nécessité de documenter les pièces, les matériaux et les instructions d'assemblage.

\section{L'évolution du projet}

\section{Le processus d'adoption de la technologie}

Chaque activité de laboratoire est perfectionnée d'une manière itérative, en réalisant des projets pilotes avec un étudiant à la fois et en apportant les ajustements nécessaires au protocole écrit. À la suite d'une seconde série d'itérations, réalisées cette fois en groupe-classe, l'activité est mise à la disposition d'autres enseignants en vue de son adoption. Les collègues du même département de physique que l'initiateur du projet ont été les premiers à adopter l'activité. Ils ont été suivis par les enseignants du programme de technologies du génie au même établissement.

Après une période de maturation, le protocole de laboratoire a été progressivement offert à d'autres collèges en vue d'une adoption extramurale. Diverses variantes et améliorations sont apportées à l'activité de laboratoire au fil de son utilisation. Ces améliorations deviennent de nouvelles ressources à partager au sein du réseau des établissements participants, ce qui 
contribue au bénéfice collectif du projet. Le tableau 3 résume l'historique de l'utilisation des deux exemples de laboratoires décrits ci-dessus.

Tableau 3

Historique de l'utilisation des activités de laboratoire

\begin{tabular}{|c|c|c|c|c|c|c|}
\hline Année & Trimestre & Activité & Département & Collège & $\begin{array}{l}\text { Nombre } \\
\text { de classes }\end{array}$ & $\begin{array}{l}\text { Nombre } \\
\text { d'étudiants }\end{array}$ \\
\hline \multirow[t]{2}{*}{2017} & Automne & TiltTray & Physique & John Abbott & 0 & 4 \\
\hline & & TiltTray & Physique & John Abbott & 1 & 40 \\
\hline \multirow[t]{4}{*}{2018} & Hiver & SpinFrame & Physique & John Abbott & 0 & 3 \\
\hline & & TiltTray & Physique & John Abbott & 4 & 160 \\
\hline & & SpinFrame & Technologies du génie & John Abbott & 1 & 25 \\
\hline & & TiltTray & Technologies du génie & John Abbott & 1 & 25 \\
\hline \multirow[t]{7}{*}{2018} & Automne & SpinFrame & Physique & John Abbott & 0 & 1 \\
\hline & & SpinFrame & Physique & John Abbott & 4 & 160 \\
\hline & & SpinFrame & Physique & Marianopolis & 0 & 1 \\
\hline & & SpinFrame & Physique & Marianopolis & 1 & 30 \\
\hline & & TiltTray & Physique & John Abbott & 0 & 1 \\
\hline & & TiltTray & Physique & Marianopolis & 1 & 30 \\
\hline & & TiltTray & Physique & John Abbott & 7 & 280 \\
\hline \multirow[t]{5}{*}{2019} & Hiver & SpinFrame & Physique & Marianopolis & 11 & 330 \\
\hline & & SpinFrame & Technologies du génie & John Abbott & 1 & 25 \\
\hline & & TiltTray & Technologies du génie & John Abbott & 1 & 25 \\
\hline & & TiltTray & Physique & Marianopolis & 3 & 90 \\
\hline & & TiltTray & Physique & John Abbott & 4 & 160 \\
\hline \multirow[t]{4}{*}{2019} & Automne & SpinFrame & Design industriel* & Vieux Montréal & 1 & $15^{*}$ \\
\hline & & TiltTray & Physique & John Abbott & 4 & 160 \\
\hline & & SpinFrame & Physique & Marianopolis & 8 & 240 \\
\hline & & SpinFrame & Physique & John Abbott & 4 & 160 \\
\hline \multirow[t]{9}{*}{2020} & Hiver & SpinFrame & Technologies du génie & John Abbott & 1 & 25 \\
\hline & & TiltTray & Technologies du génie & John Abbott & 1 & 25 \\
\hline & & TiltTray & Physique & John Abbott & 1 & 40 \\
\hline & & TiltTray & Sciences de la nature* & Vieux Montréal & 2 & 41 \\
\hline & & SpinFrame & Sciences de la nature* & Vieux Montréal & 2 & 41 \\
\hline & & TiltTray & Génie mécanique & Vieux Montréal & 1 & $17 *$ \\
\hline & & SpinFrame & Génie mécanique & Vieux Montréal & 1 & $17 *$ \\
\hline & & SpinFrame & Physique & Marianopolis & 11 & $340 * *$ \\
\hline & & & & Total & 77 & $2171^{*}$ \\
\hline
\end{tabular}

* Une valeur erronée figurait dans une première version de ce tableau, diffusée jusqu'à la mi-octobre 2020.

** Cet élément a été annulé en raison de la pandémie de COVID-19. Il est exclu du total général. 


\section{L'élargissement du répertoire des activités de laboratoire}

Les deux activités décrites ci-dessus couvrent les sujets classiques du plan incliné et du mouvement circulaire. D'autres activités de laboratoire utilisant les mêmes outils numériques sont en développement. Ces expériences portent sur l'oscillation, le développement du raisonnement spatial aux fins de la compréhension des phénomènes magnétiques, ainsi que l'identification de symboles alphabétiques tracés à la main à partir des signaux d'un accéléromètre.

\section{La capacité d'impression 3D dans le réseau collégial}

Le recours à la technologie d'impression 3D pour concevoir et produire l'équipement d'appoint nécessaire aux activités de laboratoire ouvre un tout nouveau cadre stimulant de partage et de collaboration entre les collèges. Les établissements participants peuvent désormais télécharger et imprimer non seulement le protocole d'une activité de laboratoire (sur papier), mais aussi l'équipement que les étudiants vont manipuler au cours de cette activité (en 3D). Une trousse de ressources en libre accès contient tous les documents nécessaires (tableau 4).

Tableau 4

Éléments de la trousse de ressources d'enseignement en libre accès

\begin{tabular}{ll}
\hline Catégorie & Élément \\
\hline Activités de laboratoire & Protocoles de laboratoire à l'intention des étudiants \\
& Marche à suivre pour installer et utiliser l'appli \\
& Notes pédagogiques (durée, compétences, stratégies) \\
& Matériel de présentation des accéléromètres \\
\hline Équipement imprimable en 3D & Fichiers sources de modélisation 3D \\
& Fichiers d'impression 3D (en format universel STL) \\
& Guide d'impression 3D \\
& Guide de construction (liste des pièces, matériaux, instructions d'assemblage) \\
\hline
\end{tabular}

Une telle vision prospective n'aurait pas été possible sans l'orientation résolument technologique du collège hôte du projet ${ }^{2}$. D'une part, l'établissement avait déjà investi dans une douzaine d'imprimantes 3D pour son programme de technologies du génie, dont une imprimante à grand volume; d'autre part (un aspect à ne pas négliger), ses techniciens de laboratoire avaient acquis des compétences dans le calibrage et l'entretien de cet équipement, ainsi que dans le soutien à son utilisation par les étudiants et le personnel enseignant.

En effet, ce nouveau modèle de prestation (protocole sur papier et équipement produit sur imprimante 3D) suppose un certain niveau de capacité d'impression $3 D$ au sein des établissements participants, ce qui comprend des investissements à la fois dans l'équipement d'impression 3D et dans la formation du personnel en vue d'une utilisation efficace de cet équipement. Une telle capacité commence à peine à émerger dans le réseau collégial. Quatre ans après le début du projet, tout l'équipement est encore produit dans le parc d'imprimantes 3D de l'établissement hôte et prêté sur une base temporaire aux premiers établissements participants.

2. Le collège John Abbott, à Sainte-Anne-de-Bellevue (Québec). 
Des essais préliminaires d'impression 3D ont eu lieu dans trois autres collèges du réseau ${ }^{3}$, mais aucun de ceux-ci ne possède encore la capacité nécessaire pour produire, à son usage exclusif, un ensemble pouvant accommoder une classe entière.

\section{Discussion}

\section{Les investissements dans l'infrastructure : une relation symbiotique}

Comme nous l'avons vu précédemment, les progrès du volet «impression $3 \mathrm{D}$ » du projet et, partant, ceux du projet dans son ensemble, dépendent du niveau de capacité d'impression 3D au sein du réseau collégial. Bien que cette capacité n'ait fait l'objet d'aucune évaluation systématique, l'expérience du projet en cours indique qu'elle est généralement lacunaire et qu'en ce sens, le projet paraît être déplorablement en avance sur son époque.

Or, le récent déploiement du Plan d'action numérique ouvre des perspectives tout à fait différentes sur cette situation. L'orientation 3 du Plan (tableau 1), qui représente la majeure partie du financement prévu, a trait aux investissements infrastructurels dans l'ensemble du système éducatif, sagement combinés à la liberté donnée à chaque établissement de faire des choix stratégiques dans leurs achats d'équipements. Ainsi, les collèges peuvent choisir d'investir dans le renforcement de leur capacité d'impression 3D.

Cette stratégie offre le potentiel de produire une précieuse relation symbiotique. Dans le cas du projet à l'étude, elle lève l'obstacle à l'adoption des activités de laboratoire. En effet, le projet devient à la fois attrayant et peu coûteux dès lors qu'il est possible d'imprimer localement à la fois le protocole et l'équipement de laboratoire. Ainsi, les architectes du Plan d'action numérique ont l'assurance que les choix d'investissement contribueront à des utilisations visibles et avantgardistes de la technologie en classe, tout en garantissant une égalité d'accès à tous les établissements de son territoire équipés pour l'impression 3D, même les plus isolés géographiquement.

Du point de vue des administrateurs, le projet est un exemple rare et opportun d'utilisation d'une partie des investissements infrastructurels de leur établissement à une fin à la fois tangible et visible. Il produit une image concrète de l'utilisation d'équipements de laboratoire produits au sein de l'établissement, au bénéfice d'étudiants motivés qui étudient la physique par le truchement d'un capteur de leur téléphone intelligent.

\section{La capacité d'impression 3D: de nouvelles compétences pour le personnel enseignant et de soutien}

La capacité d'impression 3D va bien au-delà de la simple acquisition d'équipement. Le personnel enseignant et le technicien de laboratoire doivent, d'une part, apprendre à utiliser le logiciel de modélisation 3D qui sert à paramétrer les objets à imprimer et, d'autre part, se familiariser avec le flux de production, des formats de fichiers de modélisation 3D jusqu'au produit final : l'objet imprimé. Il est notamment essentiel que le personnel de soutien sache calibrer et entretenir l'équipement et qu'il soit en mesure de gérer les diverses demandes d'impression ainsi que l'approvisionnement en filaments d'impression 3D.

3. Le collège Marianopolis (physique), le cégep du Vieux Montréal (design industriel) et le collège Dawson (physique). 
Les administrateurs doivent veiller à ce que leur investissement profite efficacement à une pluralité de parties prenantes et, autant que possible, trouver des moyens d'en faire un instrument de collaboration entre les départements et entre les catégories d'employés. De fait, dans notre étude de cas, dans trois des collèges qui participent actuellement au projet, des enseignants collaborent étroitement avec les techniciens de laboratoire au développement de la capacité d'impression 3D de l'établissement. Le projet a également stimulé des collaborations significatives entre les départements de physique et de technologies du génie dans un des collèges ${ }^{4}$, et entre les départements de physique et de design industriel dans un autre établissement ${ }^{5}$.

Par conséquent, le projet peut servir :

i) de stimulus pour l'adaptation et l'intégration du personnel au nouveau flux de production qu'implique la technologie d'impression 3D;

ii) d'exemple concret d'un projet exhaustif et réfléchi d'impression $3 \mathrm{D}$, orienté sur la pédagogie;

iii) de modèle et de stimulant créatif pour des projets ultérieurs dans ce type d'établissements d'enseignement.

\section{Le Plan d'action numérique : un cadre de référence}

Dans cette étude de cas, nous avons une situation intéressante: un type d'innovation (l'équipement produit par impression 3D) est développé en appui à une autre innovation (des activités de laboratoire utilisant un capteur de téléphone intelligent). Ces deux innovations ont été développées de façon parallèle et interdépendante, ce qui a donné lieu à des interactions significatives, mais elles suivent le même processus en deux phases générales, comme la plupart des projets d'innovation pédagogique.

Au cours de la phase d'établissement, l'innovation débute par des idées et des esquisses; elle est élaborée sous forme de prototype; elle fait l'objet d'essais et d'améliorations itératifs dans le cadre de programmes pilotes avec un étudiant à la fois, puis, en classe, avec un enseignant à la fois. $\mathrm{Au}$ cours de la phase d'élaboration, l'innovation est présentée à d'autres praticiens, généralement dans le même département ou le même collège que l'initiateur du projet. Un jalon important est atteint lorsque des praticiens d'autres établissements que le collège initiateur adoptent l'innovation. Ces adoptions extramurales (tableau 3) témoignent à leur tour de la valeur de l'innovation et indiquent que le projet a atteint un niveau de maturité permettant d'envisager un déploiement élargi au sein du réseau d'enseignement collégial.

Chaque phase de développement met en jeu de nouvelles priorités pour le développement ultérieur du projet. Au cours de la première phase, l'accent se porte sur la mise à disposition des ressources nécessaires aux enseignants pour réaliser l'activité de laboratoire elle-même. Au cours de la deuxième phase, la nécessité de soutenir le développement des compétences en impression 3D du personnel enseignant et de soutien forme un goulet d'étranglement pour l'élaboration ultérieure. Ces deux préoccupations correspondent aux deux catégories de ressources indiquées au tableau 4. Au cours de la phase de déploiement élargi, le goulet

4. Le collège John Abbott.

5. Le cégep du Vieux Montréal. 
d'étranglement a trait, comme nous l'avons vu, à la disponibilité de l'équipement d'impression 3D dans les collèges participants.

Il est instructif d'interpréter ces défis relatifs au développement dans l'optique du cadre établi par le Plan d'action numérique. En effet, comme l'indique le tableau 5, il est clair que le Plan d'action numérique est assez exhaustif pour prendre en compte toutes les catégories d'appui relevées dans la présente discussion.

Tableau 5

Mesures du Plan d'action numérique pertinentes pour l'étude de cas

\begin{tabular}{|c|c|c|c|c|c|}
\hline \multirow[t]{2}{*}{ Sujet général } & \multirow[t]{2}{*}{ Mesure spécifique } & \multicolumn{4}{|c|}{ Référence } \\
\hline & & $\begin{array}{l}\text { Orien- } \\
\text { tation }\end{array}$ & $\begin{array}{l}\text { Axe } \\
\text { d'inter- } \\
\text { vention }\end{array}$ & Objectif & Mesure \\
\hline \multirow[t]{3}{*}{$\begin{array}{l}\text { Développement d'un } \\
\text { projet pédagogique }\end{array}$} & $\begin{array}{l}\text { Valoriser les pratiques pédagogiques innovantes et } \\
\text { le potentiel du numérique en contexte éducatif }\end{array}$ & 1 & 2 & 1.3 & 08 \\
\hline & $\begin{array}{l}\text { Soutenir l'acquisition et le développement de } \\
\text { ressources éducatives numériques }\end{array}$ & 2 & 3 & 2.1 & 11 \\
\hline & $\begin{array}{l}\text { Encourager des projets d'innovation liés aux } \\
\text { technologies numériques }\end{array}$ & 2 & 3 & 2.1 & 12 \\
\hline $\begin{array}{l}\text { Acquisition } \\
\text { d'équipement }\end{array}$ & $\begin{array}{l}\text { Soutenir l'acquisition d'équipement numérique à } \\
\text { des fins pédagogiques dans les établissements }\end{array}$ & 3 & 8 & 3.3 & 29 \\
\hline \multirow[t]{3}{*}{$\begin{array}{l}\text { Développement des } \\
\text { compétences du } \\
\text { personnel enseignant } \\
\text { et de soutien }\end{array}$} & $\begin{array}{l}\text { Produire un nouveau référentiel de compétences } \\
\text { de la profession enseignante pour favoriser } \\
\text { l'intégration des technologies numériques dans les } \\
\text { pratiques pédagogiques des futurs membres du } \\
\text { personnel enseignant }\end{array}$ & 1 & 2 & 1.2 & 04 \\
\hline & $\begin{array}{l}\text { Favoriser la formation continue du personnel } \\
\text { enseignant, professionnel et de soutien en matière } \\
\text { de pédagogie numérique }\end{array}$ & 1 & 2 & 1.2 & 05 \\
\hline & $\begin{array}{l}\text { Offrir du soutien aux usagers des établissements } \\
\text { pour les appareils numériques destinés à } \\
\text { la pédagogie }\end{array}$ & 3 & 8 & 3.3 & 31 \\
\hline
\end{tabular}

\section{Une retombée prometteuse : la recherche sur les capteurs intelligents}

La technologie des petits capteurs accélérométriques à faible coût pour microsystèmes électromécaniques est encore relativement nouvelle. Les quatre années du projet ont donné lieu à maintes explorations de leur utilité pour l'analyse de mouvements très variés. Seul un petit sousensemble de ces perspectives a été considéré comme pertinent pour la présentation de concepts fondamentaux de la physique au collégial.

L'accumulation d'idées de pointe sur l'analyse du mouvement a débouché sur un nouveau projet dérivé (Larnder, 2019c), qui définit un domaine de recherche fondamentale innovant et prometteur. Ce projet, qui met à profit des techniques d'apprentissage machine pour l'interprétation des données accélérométriques, fait l'objet d'un financement indépendant de celui du projet actuel (Fonds de recherche Nature et technologies, s.d.). Les étudiants en 
bénéficient non pas dans la salle de cours, mais par leur participation individuelle à des travaux de recherche. Il est probable que d'autres contributions à la pédagogie à l'ère numérique en découleront, sous forme d'activités éducatives mettant en jeu des techniques de calcul et l'utilisation de cadres d'apprentissage machine (Larnder, 2020).

\section{Conclusion}

Les deux défis inhérents à l'innovation dans le milieu éducatif sont l'accessibilité et la longévité. Si une nouvelle approche nécessite la mise en œuvre ou l'acquisition de trop de ressources, son accessibilité sera compromise et son adoption sera restreinte. En même temps, si elle ne peut pas évoluer avec sa communauté d'utilisateurs, elle finira par disparaître, au sens de la « survie du plus apte ». Dans notre étude de cas, l'innovation pédagogique en jeu est évidemment l'ensemble des expériences de physique faisant appel au téléphone intelligent et, comme nous l'avons vu, la réponse la plus prometteuse à ce double défi se trouve dans l'exploitation à part entière de la technologie d'impression 3D.

Le ministère de l'Éducation et de l'Enseignement supérieur du Québec (2020) reconnaît l'importance de ces défis par son appui direct à l'innovation elle-même ${ }^{6}$ et à son mécanisme de sauvegarde ${ }^{7}$. On s'attend à ce que le récent déploiement de son vaste Plan d'action numérique (MEES, 2018) procure un avantage indirect supplémentaire au projet, au moment précis où celui-ci devient prêt pour une adoption élargie dans le réseau collégial. Ces synergies résultent directement de l'exhaustivité du cadre du Plan et de l'accent qu'il met sur une gouvernance flexible et collaborative, qui accorde à chaque établissement une autonomie considérable pour profiter des occasions qui se présentent.

Notre étude de cas démontre le potentiel de la technologie d'impression 3D comme outil d'autonomisation pour l'ensemble de la communauté collégiale et plus particulièrement pour les étudiants et les enseignants, qui peuvent ainsi maîtriser la création de l'appareil qui favorisera le mieux l'apprentissage. Les licences de libre accès favorisent une saine culture de la collaboration et de l'innovation dont tout le réseau collégial pourra bénéficier. Quant au projet pris dans son ensemble, il peut servir de modèle pour inspirer des innovations similaires aux niveaux secondaire et universitaire.

En ce qui concerne les initiatives de transition numérique, nous avons un double défi à relever, particulièrement dans l'enseignement des sciences: nous avons besoin d'une transition au chapitre non seulement des outils d'apprentissage et de communication servant à présenter la matière du curriculum, mais aussi de la transformation du contenu du curriculum lui-même. Cette dernière transformation revêt une importance vitale si nous voulons préparer nos étudiants à relever les défis du monde de demain, où ils seront à la fois des utilisateurs de technologies et des scientifiques bien au fait du fonctionnement de ces technologies et, par conséquent, en mesure de concevoir d'autres innovations.

6. Le projet «Laboratoires de physique collégiale avec accéléromètres mobiles », financé par l’Entente CanadaQuébec relative à l'enseignement dans la langue de la minorité et à l'enseignement des langues secondes (ECQ).

7. Les projets «Laboratoires de physique partagés par le biais d'impression 3D » et «Élaboration d'un projet de laboratoires de physique avec accéléromètres mobiles. » financés aussi par le ECQ. 


\section{Remerciements}

Ces travaux ont bénéficié d'un appui financier dans le cadre de l'Entente Canada-Québec relative à l'enseignement dans la langue de la minorité et à l'enseignement des langues secondes.

\section{Références}

Alvermann, D. E. (2004). Media, information communication technologies, and youth literacies: A cultural studies perspective. American Behavioral Scientist, 48(1), 78-83. https://doi.org/10.1177/0002764204267271

Arribas, E., Escobar, I., Suarez, C. P., Najera, A. et Beléndez, A. (2015). Measurement of the magnetic field of small magnets with a smartphone: A very economical laboratory practice for introductory physics courses. European Journal of Physics, 36(6), article 065002. https://doi.org/10.1088/0143-0807/36/6/065002

Blazer, C. (2008). Literature review: Educational technology (ED536868). ERIC. https://eric.ed.gov/?id=536868

Buck, J. L., McInnis, E. et Randolph, C. (2013). The new frontier of education: The impact of smartphone technology in the classroom. Dans Proceedings of the 2013 ASEE Southeast Section Conference. http://se.asee.org/...

Crompton, H., Burke, D., Gregory, K. H. et Gräbe, C. (2016). The use of mobile learning in science: A systematic review, Journal of Science Education and Technology, 25(2), 149-160. https://doi.org/10.1007/s10956-015-9597-x

Egri, S. et Szabó, L. (2015). Analyzing oscillations of a rolling cart using smartphones and tablets. The Physics Teacher, 53(3), 162-164. https://doi.org/10.1119/1.4908086

Fonds de recherche Nature et Technologies. (s.d.). Inférence de mouvements contraints à partir d'un signal d'accéléromètre - Christopher Isaac Larnder. Résumé du projet. Gouvernement du Québec. http://frqnt.gouv.qc.ca/...

Gardner, J. W., Varadan, V. K. et Awadelkarim, O. O. (2001). Microsensors, MEMS, and smart devices. John Wiley \& Sons. https://doi.org/10.1002/9780470846087

Hinrichsen, P. F. et Larnder, C. I. (2018). Combined viscous and dry friction damping of oscillatory motion. American Journal of Physics, 86(8), 577-584.

https://doi.org/10.1119/1.5034345

Hochberg, K., Kuhn, J. et Müller, A. (2016). Science education with handheld devices: A comparison of Nintendo WiiMote and iPod touch for kinematics learning. Perspectives in Science, 10, 13-18. https://doi.org/10.1016/j.pisc.2016.01.008

Langan, D., Schott, N., Wykes, T., Szeto, J., Kolpin, S., Lopez, C. et Smith, N. (2016). Students' use of personal technologies in the university classroom: Analysing the perceptions of the digital generation. Technology, Pedagogy and Education, 25(1), 101-117. https://doi.org/10.1080/1475939X.2015.1120684

Larnder, C. I. (2019a). Acceleration discontinuities in dry-friction oscillations. American Journal of Physics, 87(10), 784. https://doi.org/10.1119/1.5123455 
Larnder, C. I. (2019b, 12 avril). L'impression 3D pour des expériences de physique exploitant les téléphones intelligents [3D-print technology for smartphone-based physics experiments]. Profweb. https://profweb.ca/...

Larnder, C. I. (2020, mai). Enseigner les sciences dans un cadre d'apprentissage automatique [communication]. $7^{\mathrm{e}}$ colloque international en Éducation. enjeux actuels et futurs de la formation et de la profession enseignante, Montréal, Canada. https://colloque2020.crifpe.ca/fr/papers/details/704

Larnder, C. I. et Portelance, E. (2019a, 30 janvier). Impression 3D : quel potentiel pour les laboratoires de physique? [vidéo]. Webinaire tenu par l'Association pour les applications pédagogiques de l'ordinateur au postsecondaire (APOP). https://apop.qc.ca/...

Larnder, C. I. et Portelance, E. (2019b, mai). La fabrication additive distribuée au service de l'éducation scientifique : l'étude de cas des laboratoires de physique basés sur l'utilisation des téléphones intelligents [résumé de communication]. Colloque $603-$ Enjeux globaux et locaux de l'innovation technologique et sociale : réalités et potentiels des Living Labs, Fab Labs et labos citoyens. Congrès annuel de l'Association francophone pour le savoir (ACFAS), Ottawa, Canada. http://acfas.ca/...

Mammadova, T. (2018). Smartphones and their role in the modern classroom. International Journal of Technologies in Higher Education, 15(2), 5-14. https://doi.org/10.18162/ritpu-2018-v15n2-01

Ministère de l'Éducation et de l'Enseignement supérieur. (2018). Plan d'action numérique en éducation et en enseignement supérieur. Les élèves et les étudiants : au cour de la révolution numérique. Gouvernement du Québec. http://education.gouv.qc.ca/...

Ministère de l'Éducation et de l'Enseignement supérieur. (2020). Entente Canada-Québec. Projets soutenus financièrement. Gouvernement du Québec. http://education.gouv.qc.ca/...

Monteiro, M. et Martí, A. C. (2016). Using smartphone pressure sensors to measure vertical velocities of elevators, stairways, and drones. Physics Education, 52(1), article 015010. https://doi.org/10.1088/1361-6552/52/1/015010

Moon, R. W. (2018, 19 septembre). Apprendre la physique en accéléré avec un téléphone intelligent [Accelerated learning with smartphones in physics]. Profweb. https://profweb.ca/...

Morley, D. (2001). Belongings: Place, space and identity in a mediated world. European Journal of Cultural Studies, 4(4), 425-448. https://doi.org/10.1177/136754940100400404

Schindler, L. A., Burkholder, G. J., Morad, O. A. et Marsh, C. (2017). Computer-based technology and student engagement: A critical review of the literature. International Journal of Educational Technology in Higher Education, 14(1), article 25. https://doi.org/10.1186/s41239-017-0063-0

Strimel, G. et Grubbs, M. E. (2016). Positioning technology and engineering education as a key force in STEM education. Journal of Technology Education, 27(2), 21-36. https://doi.org/10.21061/jte.v27i2.a.1

Zwicker, A. Bloom, J., Albertson, R. et Gershman, S. (2015). The suitability of 3D printed plastic parts for laboratory use. American Journal of Physics, 83(3), 281-285. https://doi.org/10.1119/1.4900746 